Max-Planck-Institut für demografische Forschung

Max Planck Institute for Demographic Research

Doberaner Strasse 114 - D-18057 Rostock - GERMANY

Tel +49 (0) 3812081 - 0; Fax +49 (0) 3812081 - 202;

http://www.demogr.mpg.de

MPIDR WORKING PAPER WP 2002-013

FEBRUARY 2002 (REVISED SEPTEMBER 2002)

\title{
Genetic analysis of cause of death \\ in a bivariate lifetime model with dependent competing risks
}

\author{
Andreas Wienke (wienke@ demogr.mpg.de) \\ Kaare Christensen (kchristensen@ health.sdu.dk) \\ Axel Skytthe (askytthe@ health.sdu.dk) \\ Anatoli I. Yashin (yashin@demogr.mpg.de)
}

This working paper has been approved for release by: Anatoli Yashin (yashin@ demogr.mpg.de),

Head of the Laboratory of Advanced Statistical Methods.

(C) Copyright is held by the authors.

Working papers of the Max Planck Institute for Demographic Research receive only limited review.

Views or opinions expressed in working papers are attributable to the authors and do not necessarily reflect those of the Institute. 


\section{Abstract}

A mixture model in multivariate survival analysis is presented, whereby heterogeneity among subjects creates divergent paths for the individual's risk of experiencing an event (i.e., disease), as well as for the associated length of survival. Dependence among competing risks is included and rendered testable. This method is an extension of the bivariate correlated gamma-frailty model. It is applied to a data set on Danish twins, for whom cause-specific mortality is known. The use of multivariate data solves the identifiability problem which is inherent in the competing risk model of univariate lifetimes. We analyse the influence of genetic and environmental factors on frailty. Using a sample of 1470 monozygotic (MZ) and 2730 dizygotic (DZ) female twin pairs, we apply five genetic models to the associated mortality data, focusing particularly on death from coronary heart disease (CHD). Using the best fitting model, the inheritance risk of death from CHD was 0.39 (standard error 0.13 ). The results from this model are compared with the results from earlier analysis that used the restricted model, where the independence of competing risks was assumed. Comparing both cases, it turns out, that heritability of frailty on mortality due to CHD change substantially. Despite the inclusion of dependence, analysis confirms the significant genetic component to an individual's risk of mortality from CHD. Whether dependence or independence is assumed, the best model for analysis with regard to CHD mortality risks is an AE model, implying that additive factors are responsible for heritability in susceptibility to CHD. The paper ends with a discussion of limitations and possible further extensions to the model presented.

\section{Introduction}

Many studies of genetic epidemiology focus on the analysis of binary phenotypic traits such as the presence or absence of a particular disease. There is often, however, additional data such as information concerning the interval of time before the onset of the disease that is not included in such studies. In order to successfully incorporate all such useful information, it is necessary to combine models of survival analysis with models of epidemiology. Survival analysis models enhance the researcher's ability to handle censored and truncated data. Recent papers treating genetic analysis of time periods of events have been divided in their conclusions. One camp suggests the use of 'liability' models of survival (Neale et al. 1989; Meyer et al. 1991) while another has focused on 'frailty' models (Clayton and Cuzick 1985; Hougaard et al. 1992; Vaupel 1988; Yashin and Iachine 1995). Bivariate frailty models have provided an especially powerful analytic 
tool for managing identifiability problems within univariate approaches (Tsiatis 1975). All of the above-mentioned models are flawed in that they do not take into account specific causes of death. The problem with this is that the importance of genetic factors varies with each disease. Genetic epidemiology seeks to discover the association between genes and diseases. It might be useful to examine the genetic components of the susceptibility to specific diseases and death rather than to longevity. For this purpose we have extended the correlated gamma-frailty model of Yashin and Iachine (1995), which takes into account the life-spans of related individuals in order to better estimate the effect of genetic factors influencing frailty and morbidity. This approach, in our case using Danish twin females, allows us to combine lifespan data with morbidity data.

Recently, we analysed cause-specific mortality data using the correlated gamma-frailty model, assuming independence among causes of death in a 'competing risk' scenario (Wienke et al. 2000, 2001). In this paper, we investigate the effect of removing this limitation. The model allows us to test the hypothesis on dependence between the competing risks. The class of multivariate distributions presented is characterized by the association parameters, using arbitrary marginal distributions. The multivariate distribution is specified in full by the association and variance parameters and the marginal distribution functions.

We can empirically demonstrate the advantages of this new model, having revisited the statistical analysis of the lifespan data previously explored in Wienke et al. (2000, 2001). In this analysis, we focused on the mortality rates of coronary heart disease (CHD). To simplify description, in this paper we consider models limited to two competing risks (death as a result of CHD and death arising from other causes). The model can be extended to the case of multiple competing risks or multivariate lifetimes. Results of a simulation study are included. Both limitations and future uses for this model are discussed.

\section{$2 \quad$ Statistical Model}

Identifying correlations of durations is a requirement for successfully analysing genetic factors. In survival analysis there is a recurring problem of censored data, which complicates observations far more than does complete data. Using a survival model to estimate correlations among lifetimes can solve this problem. In this paper, instead of treating life spans directly, we wish to analyse both genetic and environmental factors acting on frailty for cause-specific mortality. The correlated gamma-frailty model can be used to fit the lifetime data and provide a specific parameter for the correlation among frailties. 
Let $\left(X_{11}, X_{21}\right), \ldots,\left(X_{1 n}, X_{2 n}\right)$ be independent and identically distributed (i.i.d.) nonnegative two-dimensional random vectors (pairs of lifetimes). The bivariate lifetimes $\left(X_{1 j}, X_{2 j}\right)(j=1, \ldots, n)$ are censored from the right by i.i.d. pairs of non-negative random variables $\left(C_{11}, C_{21}\right), \ldots,\left(C_{1 n}, C_{2 n}\right)$ independent of the $\left(X_{1 j}, X_{2 j}\right)$. Thus, instead of $\left(X_{1 j}, X_{2 j}\right)$ we observe $\left(T_{1 j}, T_{2 j}, \Delta_{1 j}, \Delta_{2 j}\right)$ with $T_{i j}=\min \left\{X_{i j}, C_{i j}\right\}, \Delta_{i j}=1\left(X_{i j} \leq C_{i j}\right)$ $(i=1,2 ; j=1, \ldots, n)$ where $1(\cdot)$ denotes the indicator function of the event in the brackets.

Let us assume that the lifetimes follow a distribution given by the survival function $S\left(x_{1}, x_{2}\right)=P\left(X_{1 j}>x_{1}, X_{2 j}>x_{2}\right)$ and denote by $C\left(c_{1}, c_{2}\right)=P\left(C_{1 j}>c_{1}, C_{2 j}>c_{2}\right)$ the survival function of censoring times. Hence, the survival function of the four-dimensional non-observable data is

$$
S\left(x_{1}, c_{1}, x_{2}, c_{2}\right)=S\left(x_{1}, x_{2}\right) C\left(c_{1}, c_{2}\right) .
$$

This form is a consequence of the independence between lifetimes and censoring times. Furthermore, due to the structure of the data we will be using as an example, let us assume that both lifetimes in each pair are left truncated at the same time $w_{j}$, which is common in twin studies. (Note that it is not a problem to deal with different truncation times in other pairs of relatives.) Consequently, observable data $\left(T_{1 j}^{*}, T_{2 j}^{*}, \Delta_{1 j}^{*}, \Delta_{2 j}^{*}, w_{j}\right)$ have a conditional distribution of the form

$$
\mathcal{L}\left(T_{1 j}^{*}, T_{2 j}^{*}, \Delta_{1 j}^{*}, \Delta_{2 j}^{*}, w_{j}\right)=\mathcal{L}\left(T_{1 j}, T_{2 j}, \Delta_{1 j}, \Delta_{2 j} \mid T_{1 j}>w_{j}, T_{2 j}>w_{j}\right) .
$$

Here $\mathcal{L}(X)$ denotes the distribution of the random variable $X$. With this model we derive the likelihood function of the bivariate left truncated and bivariate right censored data in (2), which is given by

$$
\begin{aligned}
L\left(t_{1}, t_{2}, \delta_{1}, \delta_{2}, w\right) & =\left(\delta_{1} \delta_{2} S_{t_{1} t_{2}}\left(t_{1}, t_{2}\right)-\delta_{1}\left(1-\delta_{2}\right) S_{t_{1}}\left(t_{1}, t_{2}\right)\right. \\
& \left.-\left(1-\delta_{1}\right) \delta_{2} S_{t_{2}}\left(t_{1}, t_{2}\right)+\left(1-\delta_{1}\right)\left(1-\delta_{2}\right) S\left(t_{1}, t_{2}\right)\right) / S(w, w) .
\end{aligned}
$$

Here $\left(t_{1}, t_{2}, \delta_{1}, \delta_{2}, w\right)$ denotes a realisation of the random vector $\left(T_{1 j}^{*}, T_{2 j}^{*}, \Delta_{1 j}^{*}, \Delta_{2 j}^{*}, w_{j}\right)$. Partial derivatives of the marginal survival functions are given by $S_{t_{i}}\left(t_{1}, t_{2}\right)=\frac{\partial S\left(t_{1}, t_{2}\right)}{\partial t_{i}}(i=$ $1,2)$ and $S_{t_{1} t_{2}}\left(t_{1}, t_{2}\right)=\frac{\partial S\left(t_{1}, t_{2}\right)}{\partial t_{1} \partial t_{2}}$. Because of the independence of lifetimes $\left(X_{1 j}, X_{2 j}\right)$ and censoring times $\left(C_{1 j}, C_{2 j}\right)(j=1, \ldots, n)$ the distribution of the censoring times does not enter the likelihood function. Assuming a correlated gamma-frailty model for the survival times such that:

$$
S\left(x_{1}, x_{2}\right)=\frac{S\left(x_{1}\right)^{1-\rho} S\left(x_{2}\right)^{1-\rho}}{\left(S\left(x_{1}\right)^{-\sigma^{2}}+S\left(x_{2}\right)^{-\sigma^{2}}-1\right)^{\frac{\rho}{\sigma^{2}}}},
$$


this model was used to describe total mortality in twins by Yashin and Iachine (1995) and cause-specific mortality in twins under the assumption of independence between competing risks by Wienke et al. (2000, 2001). Here, $S(x)=S(x, 0)=S(0, x)$ denotes the marginal survival functions, which are assumed to be equal for twins. However, the assumption of independence between competing risks is questionable. Typically, in clinical and epidemiological studies two different types of censoring occur. The observation of certain individuals are censored due to the fact that they are still alive at the end of the study. Other individuals drop from follow-up for reasons not associated with the disease under study, but through life-events beyond the control of the researcher, such as migration.

If censoring can be assumed to be non-informative with regard to all different causes, then the model above may be applied with the censoring times $\left(C_{1 j}, C_{2 j}\right)(j=1, \ldots, n)$ taken as the minimum of the hypothetical censoring times arising from the different causes of censoring. For estimating the marginal survival function $S(x)$ in (4) the KaplanMeier estimator is appropriate. However, the situation becomes much more difficult if the censoring arising from at least one of the different causes can be assumed to be informative.

In the following, we consider a case where two types of censoring occur, one noninformative and the other informative. Let $\left(X_{1 j}, Y_{1 j}, C_{1 j}, X_{2 j}, Y_{2 j}, C_{2 j}\right)(j=1, \ldots, n)$ be i.i.d. vectors of non-negative random variables. The variables $\left(X_{1 j}, X_{2 j}\right)$ denote the (usually non-observable) lifetimes (with respect to the cause of death of interest) of pairs of individuals. The $\left(Y_{1 j}, Y_{2 j}\right)$ are informative censoring times (which may be lifetimes with respect to causes of death not under study) and the $\left(C_{1 j}, C_{2 j}\right)$ are non-informative censoring times (for example caused by end of study). Again, for $j=1, \ldots, n$ and $i=1,2$ we observe $T_{i j}=\min \left\{X_{i j}, Y_{i j}, C_{i j}\right\}$ and

$$
\Delta_{i j}=\left\{\begin{aligned}
1 & : \quad \text { if } X_{i j} \leq \min \left\{C_{i j}, Y_{i j}\right\} \\
0 & : \quad \text { if } C_{i j}<\min \left\{X_{i j}, Y_{i j}\right\} \\
-1 & : \quad \text { if } Y_{i j}<\min \left\{X_{i j}, C_{i j}\right\}
\end{aligned}\right.
$$

where $\Delta_{i j}=1$ means no censoring, $\Delta_{i j}=0$ is non-informative censoring and $\Delta_{i j}=-1$ is informative censoring. Now we derive the six-dimensional survival function of the data. Suppose that we use $\left(X_{1}, Y_{1}, X_{2}, Y_{2}\right)$ as a shorthand for $\left(X_{1 j}, Y_{1 j}, X_{2 j}, Y_{2 j}\right)(j=1, \ldots, n)$. Let $\left(X_{1}, Y_{1}, X_{2}, Y_{2}\right)$ and $\left(Z_{1}, Z_{2}, Z_{3}, Z_{4}\right)$ be the survival times of life- and (informative) censoring times and the frailties of the two individuals with respect to two different causes of death; let their individual hazards be given by the proportional hazards model 
(without covariates)

$$
\begin{aligned}
& X_{1} \sim \mu_{1}\left(x_{1}, Z_{1}\right)=Z_{1} \mu_{1}\left(x_{1}\right) \quad X_{2} \sim \mu_{1}\left(x_{2}, Z_{3}\right)=Z_{3} \mu_{1}\left(x_{2}\right) \\
& Y_{1} \sim \mu_{2}\left(y_{1}, Z_{2}\right)=Z_{2} \mu_{2}\left(y_{1}\right) \quad Y_{2} \sim \mu_{2}\left(y_{2}, Z_{4}\right)=Z_{4} \mu_{2}\left(y_{2}\right),
\end{aligned}
$$

where $X \sim \mu$ means, that $\mu$ denotes the hazard function of $X$. Hence, the conditional distribution of the lifetime of the first $\left(X_{1}\right)$ and second twin $\left(X_{2}\right)$ with respect to the first cause of death are assumed equal (given by $\mu_{1}$ ). The same is true for the lifetime of the first $\left(Y_{1}\right)$ and second twin $\left(Y_{2}\right)$ with respect to the second cause of death $\left(\mu_{2}\right)$. Note, that $\mu_{i}(x, Z)=Z \mu_{i}(x)$ implies the relation $S_{i}(x \mid Z)=S_{0 i}(x)^{Z}(i=1,2)$ and $S_{0 i}$ are the survival functions related to the baseline hazard functions $\mu_{i}$. We assume that $X_{1}, Y_{1}, X_{2}, Y_{2}$ are independent given the vector of frailties $\left(Z_{1}, Z_{2}, Z_{3}, Z_{4}\right)$. Let $V_{1}, V_{8} \sim \Gamma\left(k_{1}, \lambda_{0}\right), V_{2} \sim \Gamma\left(k_{2}, \lambda_{1}\right), V_{3} \sim \Gamma\left(k_{3}, \lambda_{2}\right), V_{4}, V_{7} \sim \Gamma\left(k_{4}, \lambda_{2}\right), V_{5}, V_{6} \sim \Gamma\left(k_{5}, \lambda_{1}\right)$ independent gamma distributed random variables with parameters $k_{1}+k_{2}+k_{5}:=\lambda_{1}=\frac{1}{\sigma_{1}^{2}}$ and $k_{1}+k_{3}+k_{4}:=\lambda_{2}=\frac{1}{\sigma_{2}^{2}}$. Now the frailties are given by the following construction:

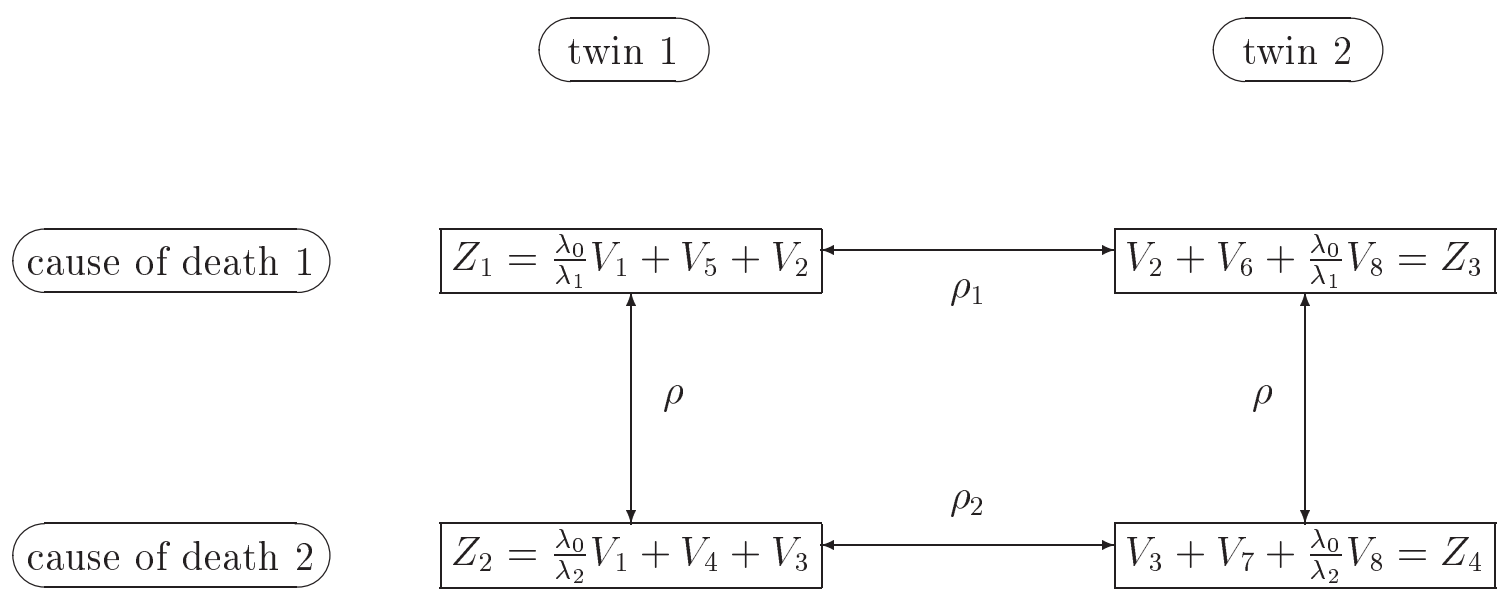

Figure 1: Cause-specific frailties and their correlations in a twin pair

Here $Z_{1}, Z_{3}$ denote frailties with respect to the first cause of death (cause under study) and $Z_{2}, Z_{4}$ denote frailties with respect to the second cause of death of both individuals. Furthermore, $\rho$ describes variously the correlations between the frailties: $\rho_{1}=$ $\operatorname{corr}\left(Z_{1}, Z_{3}\right), \rho_{2}=\operatorname{corr}\left(Z_{2}, Z_{4}\right)$ and $\rho=\operatorname{corr}\left(Z_{1}, Z_{2}\right)=\operatorname{corr}\left(Z_{3}, Z_{4}\right)$. Now the sixdimensional survival function can be derived by averaging over the conditional lifetimes, using relation (6) and applying the laplace transform of gamma distributed random 
variables (for more detailed calculations see Appendix):

$$
\begin{aligned}
S\left(x_{1}, y_{1}, c_{1}, x_{2}, y_{2}, c_{2}\right) \\
=\mathbf{E} S_{1}\left(x_{1}\right)^{Z_{1}} S_{2}\left(y_{1}\right)^{Z_{2}} S_{1}\left(x_{2}\right)^{Z_{3}} S_{2}\left(y_{2}\right)^{Z_{4}} C\left(c_{1}, c_{2}\right) \\
=\left(S_{1}\left(x_{1}\right)^{-\sigma_{1}^{2}}+S_{1}\left(x_{2}\right)^{-\sigma_{1}^{2}}-1\right)^{-\frac{\rho_{1}}{\sigma_{1}^{2}}}\left(S_{2}\left(y_{1}\right)^{-\sigma_{2}^{2}}+S_{2}\left(y_{2}\right)^{-\sigma_{2}^{2}}-1\right)^{-\frac{\rho_{2}}{\sigma_{2}^{2}}} \\
*\left(S_{1}\left(x_{1}\right)^{-\sigma_{1}^{2}}+S_{2}\left(y_{1}\right)^{-\sigma_{2}^{2}}-1\right)^{-\frac{\rho}{\sigma_{1} \sigma_{2}}}\left(S_{1}\left(x_{2}\right)^{-\sigma_{1}^{2}}+S_{2}\left(y_{2}\right)^{-\sigma_{2}^{2}}-1\right)^{-\frac{\rho}{\sigma_{1} \sigma_{2}}} \\
* S_{1}\left(x_{1}\right)^{1-\rho_{1}-\frac{\sigma_{1}}{\sigma_{2}} \rho} S_{1}\left(x_{2}\right)^{1-\rho_{1}-\frac{\sigma_{1}}{\sigma_{2}} \rho} S_{2}\left(y_{1}\right)^{1-\rho_{2}-\frac{\sigma_{2}}{\sigma_{1}} \rho} S_{2}\left(y_{2}\right)^{1-\rho_{2}-\frac{\sigma_{2}}{\sigma_{1}} \rho} C\left(c_{1}, c_{2}\right)
\end{aligned}
$$

with $0 \leq \rho \leq \min \left\{\frac{\sigma_{2}}{\sigma_{1}}\left(1-\rho_{1}\right), \frac{\sigma_{1}}{\sigma_{2}}\left(1-\rho_{2}\right)\right\}$. In this model $\rho_{1}$ denotes the correlation between $Z_{1}$ and $Z_{3}$, the main parameter of interest. This parameter describes the correlation of frailties of individuals in a pair with respect to the cause of death under study and is the key figure for genetic analysis of susceptibility to death from cause-specific mortality. The second parameter $\rho_{2}$ models the correlation between frailties with respect to all other causes of death (combined to the second cause of death or informative censoring). The parameter $\rho$ is responsible for the association between causes of death in each individual. With this parameter, it is possible to test the hypothesis of dependence between competing risks in the above model.

$S_{1}$ and $S_{2}$ are the marginal survival functions with respect to the first and second cause of death. Please note that it is impossible to use the Kaplan-Meier estimator to get non-parametric estimates of the marginal survival functions because of the assumed dependence between the two competing risks. To overcome this problem we used a parametric approach by fitting a Gamma-Gompertz model to the data, e.g. $S_{i}(x)=$ $\left(1+s_{i}^{2} \frac{\alpha_{i}}{\beta_{i}}\left(e^{\beta_{i} x}-1\right)\right)^{-\frac{1}{s_{i}^{2}}},(i=1,2)$, where $\alpha_{i}, \beta_{i}, s_{i}^{2}$ are parameters to be estimated. Again, it is necessary to account for left truncation in the data. The likelihood function of this model is given in the Appendix. Due to the assumption concerning non-informative censoring with respect to the $\left(C_{1 j}, C_{2 j}\right)$ the function $C$ does not enter the likelihood function.

\section{Quantitative Genetics of Frailty}

In twin studies, the intrapair-correlations of traits found in MZ and DZ twin pairs (we use the notation $\left.\rho_{1}(M Z), \rho_{1}(D Z)\right)$ play a key role in the analysis of genetic and environmental factors.

Using these coefficients, we considered five genetic models of frailty that correspond to five different assumptions about structure. We followed the notation of Neale and Cardon (1992) for these models. Resemblance in twins are caused by three factors (completely for MZ twins and partly for DZ twins): additive genetic factors (A), genetic dominance 
factors (D) and shared environmental factors (C). Non-shared environment (E) (including measurement errors) is (completely for MZ twins and partly for DZ twins) responsible for intrapair differences in twins. Additive genetic factors contribute twice as much to the correlation in MZ twins as DZ twins because MZ twins share all their identical genes by descent, while DZ twins (like non-twin siblings) share on average only half of their genes. Dominant genetic factors contribute four times as much to the correlation in MZ twins than DZ twins according to Mendelian theory. A shared environment (with environmental factors such as social class or parental behavior, common familiar habits such as smoking, drinking, physical exercises and diet) contributes in equal measure to the correlation between MZ and DZ twins. Higher intra-pair correlations in MZ twins indicate how important a role genetic factors play. Readers unfamiliar with the use of latent variables in structural equation modeling may wonder how it is possible to reach conclusions about the role of genetic and environmental risk factors without actually measuring them directly. As in all latent variable models, the impact of genes and environment on the susceptibility to the disease of interest is inferred from the pattern of observed correlations in relatives, which are in turn predicted by Mendelian theory.

From the estimation point of view, only three parameters could be included in the model, because there are only data about two different groups of relatives (MZ and DZ twins). More complex models need data about additional groups of relatives such as parents or offspring. Each additional group of relatives in the study allow for an additional parameter, but this point is beyond the scope of the paper. The following biometric models were fitted to the data: AE, DE, ACE, ADE and CE, the ACE model refers to the decomposition of frailty $\mathrm{Z}=\mathrm{A}+\mathrm{C}+\mathrm{E}$; the $\mathrm{CE}$ model refers to the decomposition $\mathrm{Z}=\mathrm{C}+\mathrm{E}$; $\mathrm{ADE}, \mathrm{AE}$ and DE models are defined similarly. We use the small letters $a^{2}, d^{2}, c^{2}, e^{2}$ to refer to the respective proportions of variance. For example, the relation $1=a^{2}+c^{2}+e^{2}$ corresponds to the decomposition of variance in the ACE model of frailty. Depending on the best fitting model, the proportion of variance in susceptibility due to additive $\left(a^{2}\right)$ or dominant $\left(d^{2}\right)$ genetic factors is termed heritability. Shared environmental factors and dominance factors cannot be estimated simultaneously, because they are completely confounded in the classical study where twins are reared together (Heath et al., 1989). Standard assumptions about the quantitative genetics yields in the following relations:

$$
\begin{aligned}
\rho_{1}(M Z) & =a^{2}+d^{2}+c^{2} \\
\rho_{1}(D Z) & =0.5 a^{2}+0.25 d^{2}+c^{2} \\
1 & =a^{2}+d^{2}+c^{2}+e^{2}
\end{aligned}
$$

This includes the assumption that MZ and DZ twins have the same correlation in environments (equal environment assumption). This standard assumption of the classical 
twin method is necessary for the identifiability of parameters. To combine the approach of quantitative genetics with the methods of survival analysis, we used the extended correlated gamma-frailty model with genetic and environmental components of frailty. In this approach the genetic and environmental parameters of frailty decomposition are estimated directly by the maximum likelihood method. For more detailed information about this point see Yashin and Iachine (1994). The analysis was made using the statistical software package GAUSS.

\section{Simulation}

\subsection{Simulation design}

All simulations involve generating gamma-distributed frailties, bivariate lifetimes, dependent and independent censoring times and truncation times. We will try to mimic the characteristics of the Danish twin data which we analyse in the next section. A total of 8000 twin pairs (3000 MZ and $5000 \mathrm{DZ}$ pairs) are simulated, a number which is reduced by the truncation process to a final sample size of around 4200-4300 twin pairs. Samples are generated as follows:

- Generate frailty variables $Z_{1}, Z_{2}, Z_{3}, Z_{4}$ using independent gamma-distributed random variables $V_{1}, \ldots, V_{8}$.

- Generate lifetimes with respect to the first $\left(X_{1}, X_{2}\right)$ and second disease $\left(Y_{1}, Y_{2}\right)$ given the frailties using $S_{i}(x)=\left(1+s_{i}^{2} \frac{\alpha_{i}}{\beta_{i}}\left(e^{\beta_{i} x}-1\right)\right)^{-\frac{1}{s_{i}^{2}}},(i=1,2)$.

- The censored (bivariate) lifetimes $T_{i j}=\min \left\{X_{i j}, Y_{i j}, C_{i j}\right\}$ are generated by using the lifetimes with respect to the second cause of death as dependent censoring times and uniform distributed random variables on [40,100] as independent censoring times.

- Birth years are generated by using a uniform distribution on $[1870,1930]$ to mimic the truncation pattern.

- Year of truncation is 1943.

The simulation program was written using the GAUSS language. We simulated 500 data sets. 


\subsection{Simulation results}

The mean parameter estimates of the model are shown in Table 1, in comparison with the true values used for simulation. Although there appears to be some bias in certain parameter estimates, the magnitude does not appear to be of any practical significance and the overall performance is quite accurate.

\begin{tabular}{lccc}
\hline Parameter & true value & Mean of estimates & standard deviation of estimates \\
\hline$\alpha_{1}$ & 1.000 & 1.010 & 0.278 \\
$\beta_{1}$ & 0.120 & 0.121 & 0.008 \\
$s_{1}$ & 2.000 & 1.992 & 0.202 \\
$\alpha_{2}$ & 1.000 & 0.993 & 0.311 \\
$\beta_{2}$ & 0.120 & 0.122 & 0.009 \\
$s_{2}$ & 2.000 & 2.062 & 0.250 \\
$\sigma_{1}$ & 2.000 & 1.956 & 0.287 \\
$\sigma_{2}$ & 2.000 & 2.174 & 0.684 \\
$\rho_{1}(M Z)$ & 0.400 & 0.410 & 0.073 \\
$\rho_{1}(D Z)$ & 0.200 & 0.206 & 0.050 \\
$\rho_{2}(M Z)$ & 0.100 & 0.108 & 0.064 \\
$\rho_{2}(D Z)$ & 0.060 & 0.067 & 0.051 \\
$\rho$ & 0.500 & 0.533 & 0.237 \\
\hline
\end{tabular}

Table 1: Parameter estimation in the simulation study.

\section{Example}

In our example, we investigated how well the method performed when used to analyse the respective influence of genetic and environmental factors affecting risk of mortality from coronary heart disease (CHD). In this example the second cause of death is all other causes combined. The data we use for our analysis are the survival times of MZ and DZ female twins sampled from the Danish Twin Registry, the first national twin registry world-wide (established in 1954 by Bent Harvald and Mogens Hauge). This population-based registry includes all twins born in Denmark during the period 18701910 and all same-sex pairs born between 1911 and 1930. For detailed information about the Danish Twin Registry see Hauge (1981). The data set contains records of female twin pairs born between 1 January 1870 and 31 December 1930 and both individuals were still alive on 1 January 1943. Consequently, the observations are left truncated. Pairs with at least one death before 31 December 1993 and incomplete ICD information 
or unknown zygosity were excluded. Individuals were followed up to 31 December 1993, and subjects identified as deceased after that date were classified here as alive. Total, we sampled $1470 \mathrm{MZ}$ twin pairs and $2730 \mathrm{DZ}$ twin pairs.

In addition to the lifetime data, there is documentation regarding the cause of death for all non-censored lifetimes. During the follow-up 369 deaths due to CHD occur among MZ twins and 704 deaths among DZ twins. CHD was defined as ICD code 420 (revision 6 and 7) and 410 - 414 (revision 8). Death status, age at death, and cause of death were obtained from the Central Person Register, the Danish Cause-of-death Register, the Danish Cancer Register (founded in 1942), and other public registers in Denmark. The validity of the twin register has been checked by comparing information about year of death with the nationwide Danish Cancer Register. Both registers were independent, but 99 \% agreement was found (Holm, 1983). Further data corrections increased this part to almost $100 \%$. Zygosity was determined by self reported similarities. Validations of this zygosity classification by comparing with laboratory methods (serological markers) show a misclassification rate of less than $5 \%$ (Lykken, 1978; Holm, 1983). For more detailed information about status, zygosity and cause of death in the population under study see Table 2 and 3.

\begin{tabular}{lcc}
\hline \multicolumn{1}{c}{ status } & monozygotic twins & dizygotic twins \\
\hline both twins dead & 622 & 1072 \\
one twin alive, cotwin dead & 332 & 773 \\
both twins alive & 516 & 885 \\
all pairs together & 1470 & 2730 \\
\hline
\end{tabular}

Table 2: Study population (number of pairs) by zygosity and life status.

\begin{tabular}{lcc}
\hline cause of death & monozygotic twins & dizygotic twins \\
\hline coronary heart disease & 369 & 704 \\
all causes together & 1576 & 2917 \\
alive (censored) & 1364 & 2543 \\
\hline
\end{tabular}

Table 3: Study population (number of individuals) by zygosity and cause of death.

First, we compared the ADE and DE as well as the ACE and CE models. The likelihood ratio test prefers the DE and the ACE model (Table 4). The ACE model converges to the AE model. Standard errors are not given in the ACE model since 0 is the boundary of the parametric space. A comparison of the $\mathrm{AE}$ and the DE model is impossible with respect to the likelihood ratio test because the models are not nested. According to the Akaike 
Information Criterion (AIC), the $\mathrm{AE}$ model fit the data best and gives a inheritance estimate of 0.39 , with standard error 0.13 . Using the sub-model of independent causes of death $(\rho=0$, model $(2)$ and $(4))$, the inheritance estimate was $0.58(0.14)$.

\begin{tabular}{lccccccc}
\hline & $\sigma$ & $a^{2}$ & $d^{2}$ & $c^{2}$ & $e^{2}$ & $\rho$ & Log-L \\
\hline $\mathrm{ACE}$ & $1.70(-)$ & $0.39(-)$ & & $0.00(-)$ & $0.61(-)$ & $0.45(-)$ & 22268.06 \\
$\mathrm{AE}$ & $1.70(0.21)$ & $0.39(0.13)$ & & & $0.61(0.13)$ & $0.45(0.12)$ & 22268.06 \\
$\mathrm{ADE}$ & $1.63(0.23)$ & $0.28(0.24)$ & $0.13(0.26)$ & & $0.59(0.12)$ & $0.49(0.06)$ & 22267.81 \\
$\mathrm{DE}$ & $1.63(0.24)$ & & $0.44(0.12)$ & & $0.56(0.12)$ & $0.49(0.07)$ & 22268.61 \\
$\mathrm{CE}$ & $1.80(0.26)$ & & & $0.22(0.00)$ & $0.78(0.07)$ & $0.54(0.06)$ & 22271.93 \\
\hline $\mathrm{AE}^{*}$ & $1.87(0.41)$ & $0.58(0.14)$ & & & $0.42(0.14)$ & 0.00 & 22269.24
\end{tabular}

Table 4: Results of genetic analysis of CHD. $\sigma^{2}$ - variance of frailty, $a^{2}$ - additive genetic effects, $d^{2}$ - genetic effects due to dominance, $c^{2}$ - shared environment, $e^{2}$ - non-shared environment, $\rho$ - correlation between frailties associated with competing risks, Log-L - value of the Log-Likelihood function divided by number of observations, $\mathrm{DE}^{*}-\mathrm{DE}$ model with $\rho=0$ (independent model)

\section{Discussion}

Frailty models are mixture models within survival analysis. In survival analysis, one typically has to deal with censored observations. In most applications censoring is assumed to be simply non-informative. This assumption is realistic for example in clinical studies, where patients contribute censored observations because they are still alive at the preassigned termination point of study. Some others get lost during the time of follow-up for reasons that are not related to the event under study. In such cases censoring can be assumed to be non-informative. However, in some cases this assumption is questionable, especially in cases where there competing causes of death. This paper has suggested using an extension of the bivariate correlated gamma-frailty model (Pickles et al., 1994; Yashin and Iachine, 1995) in such cases, where only a part of the censored observations is assumed to be non-informatively censored. Because competing risks can also be correlated within families and may share unobserved dependencies with the cause of interest, the standard approach, which treats competing risks as independent, could lead to biased estimates of the variance components associated with the cause of interest. Here, the frailties are modeled in terms of standard variance components for additive and dominance genetic effects and shared and unique environmental effects. This thus provides a rich class of models for analyzing this complex pattern of dependencies between family members and between causes of death. Furthermore, frailty models are 
well suited for inclusion of observed covariates into the analysis (Wienke et al., 2002). Using cause-specific mortality data of relatives (here twins) it is possible to overcome problems due to identifiability in univariate censored lifetimes as stated in Tsiatis (1975). The model we have evolved allows for dependencies among competing risks, and makes it possible to test for such dependencies. Furthermore, combining methods from survival analysis (especially from frailty models) and genetic analysis as we did, improves the genetic analysis of time-to-event data in the case of informative and non-informative censoring together as well as accounting for heterogeneity in the population. Our example is an extension of the analysis in the case of independent causes of death (Wienke et al., 2000, 2001), where deaths from other causes than the cause under study are treated as non-informative and collapsed with censored observations caused by end of study. In both cases (here called dependent and independent) the AE model is the best fitting model for CHD. This shows a certain degree of consistency in the model. Comparing both cases, it turns out, that the heritability of frailty on mortality due to CHD change substantially. Fixing the correlation of 0.45 (0.12) between frailty on mortality from CHD and frailty on mortality from other causes to zero has a impact on the heritability estimate - changing it from 0.39 (0.13) to 0.58 (0.14). Both models detected the significant influence of genetic factors. The parameter $\rho$ can be used to test the hypothesis of dependence between the competing risks. The likelihood ratio test indicates that the simpler independent model is sufficient to describe the data.

The proof of consistency and asymptotic normality of the maximum likelihood estimators is still an open problem, but our simulation results seem to point to the asymptotic validity of the proposed method.

One important limitation of the presented model should be kept in mind, the correlation coefficient between the frailties are always non-negative by construction. This restriction makes sense when comparing the lifetimes of relatives, but it is not clear that the same holds for the competing risks in an individual. On one hand, many major diseases have risk factors in common and consequently, the presence of any one of these risk factors will increase the risk of death with respect to all diseases. On the other hand, everyone dies eventually, so logically, if the risk of death from one cause is decreased the risk from another cause must be increased. Furthermore, the parameter $\rho$ is only identifiable in a 'real' multivariate case. Pairs of unrelated individuals (e.g. $\rho_{1}=\rho_{2}=0$ ) implies the univariate case, which makes the parameter $\rho$ non-identifiable. The nature of dependencies among competing risks deserves further study.

Classical twin studies are based on the important assumption that MZ and DZ twins have the same correlation in environments (equal environment assumption). This standard assumption is necessary for the identifiability of heritability i.e. so as to be able to 
interpret the difference in concordance between MZ and DZ twins as being explained in full by their difference in genetic concordance. However, without doubt, the assumption is also questionable: MZ twins are generally treated the same by their parents to a much greater extend than DZ twins by their parents. This implies an overestimation of heritability. The equal environment assumption seems to be acceptable with respect to environmental factors related to CHD.

The suggested model gives a clear illustration of how the methods of survival analysis and genetic epidemiology may be merged to improve the genetic investigation of time-toevent data. Further extensions of the model to multiple causes of death and/or multiple related lifetimes will be important in elucidating the properties of this strategy.

\section{Acknowledgements}

The authors wish to thank the Danish Twin Register for providing the data and Susann Backer for help in preparing the paper for publication. The research was partly supported by NIH/NIA grant 7PO1AG08761. 


\section{References}

[1] Christensen, K., J.W. Vaupel, N.V. Holm, and A.I. Yashin, Mortality among twins after age 6: fetal origins hypothesis versus twin method,

British Medical Journal 310 (1996) 432-436.

[2] Christensen, K., A. Wienke, A. Skytthe, N.V. Holm, J.W. Vaupel and A.I. Yashin, Cardiovascular mortality in twins and the fetal origins hypothesis,

Twin Research 5 (2001) 344-349.

[3] Clayton, D.G., and J. Cuzick, Multivariate generalizations of the proportional hazards model (with discussion),

Journal of the Royal Statistical Society, Series A, 148 (1985) 82-117.

[4] Hauge, M. 1981. The Danish Twin Register, in: S.A. Mednich, A.E. Baert and B.P. Bachmann (Eds). Prospective longitudinal research (Oxford Medical Publications, Oxford, 1981) p 217-222.

[5] Heath A.C., M.C. Neale, J.K. Hewitt, L.J. Eaves and D.W. Fulker, Testing structural equation models for twin data using LISREL, Behavior Genetics 19 (1989) 9-35.

[6] Holm N.V., The use of twin studies to investigate causes of diseases with complex etiology with a focus on cancer (PhD thesis, University of Odense, 1983).

[7] Hougaard, P., B. Harvald and N.V. Holm, Measuring the similarities between the lifetimes of adult Danish twins born between 1881-1930, JASA 87 (1992) 17-24.

[8] Meyer, J.M., L.J. Eaves, A.C. Heath and N.G. Martin, Estimating genetic influences on the age at menarche: A survival analysis approach, American Journal of Medical Genetics 39 (1991) 148-154.

[9] Neale, M.C. and L.R. Cardon, Methodology for genetic studies of twins and families (Kluwer, Dordrecht, 1992).

[10] Neale, M.C., L.J. Eaves, J.K. Hevitt, C.J. MacLean, J.M. Meyer and K.S. Kendler, Analysing the relationship between age at onset and risk to relatives, American Journal of Human Genetics 45 (1989) 226-239. 
[11] Pickles A., R. Crouchley, E. Simonoff, L.J. Eaves, J.M. Meyer, M. Rutter, J.K. Hevitt and J. Silberg, Survival Models for Developmental Genetic Data: Age of Onset of Puberty and Antisocial Behavior in Twins, Genetic Epidemiology 11 (1994) 155-170.

[12] Tsiatis, A.A., A nonidentifiability aspect of the problem of competing risks, Proceedings of the National Academy of Sciences 72 (1975) 20-22.

[13] Vaupel, J.W., Inherited frailty and longevity, Demography 25 (1988) 277-287.

[14] Wienke, A., K. Christensen, N.V. Holm and A.I. Yashin, Heritability of death from respiratory diseases: an analysis of Danish twin survival data using a correlated frailty model, in: Hasman et al., (Eds.) Medical Infobahn for Europe (IOS Press, Amsterdam, 2000) 407-411.

[15] Wienke, A., N. Holm, A. Skytthe and A.I. Yashin, The heritability of mortality due to heart diseases: a correlated frailty model applied to Danish twins.

Twin Research 4 (2001) 266-274.

[16] Wienke, A., A.M. Herskind, K. Christensen, A. Skytthe and A.I. Yashin, The heritability of mortality due to heart diseases: a correlated frailty model applied to Danish twins. Max Planck Institute for Demographic Research, Rostock, Germany, Working Paper \# 2002-003 (2002) (available at http://www.demogr.mpg.de)

[17] Yashin A.I. and I.A. Iachine, Environment determines $50 \%$ of variability in individual frailty: results from Danish twin study. Research Report, Population Studies of Aging 10 (1994), Odense University, Denmark.

[18] Yashin, A.I. and I.A. Iachine, Genetic Analysis of Durations: Correlated Frailty Model Applied to Survival of Danish Twins,

Genetic Epidemiology 12 (1995) 529-538. 


\section{Appendix}

The following relations are used in the calculations: $\mathbf{E} Z_{1}=\mathbf{E} Z_{2}=\mathbf{E} Z_{3}=\mathbf{E} Z_{4}=1$, $\mathbf{V}\left(Z_{1}\right)=\mathbf{V}\left(Z_{3}\right)=\frac{1}{k_{1}+k_{2}+k_{5}}=\sigma_{1}^{2}, \mathbf{V}\left(Z_{2}\right)=\mathbf{V}\left(Z_{4}\right)=\frac{1}{k_{1}+k_{3}+k_{4}}=\sigma_{2}^{2}$.

The second moment of $V_{2}$ is needed to calculate the mixed moment $\mathbf{E} Z_{1} Z_{3}$ :

$$
\mathbf{E} V_{2}^{2}=\mathbf{V}\left(V_{2}\right)+\left(\mathbf{E} V_{2}\right)^{2}=\frac{k_{2}}{\lambda_{1}^{2}}+\left(\frac{k_{2}}{\lambda_{1}}\right)^{2}=\frac{k_{2}^{2}+k_{2}}{\lambda_{1}^{2}}
$$

Hence,

$$
\begin{aligned}
\mathbf{E} Z_{1} Z_{3} & =\mathbf{E}\left(\frac{\lambda_{0}}{\lambda_{1}} V_{1}+V_{2}+V_{5}\right)\left(V_{2}+V_{6}+\frac{\lambda_{0}}{\lambda_{1}} V_{8}\right) \\
& =\mathbf{E}\left(\frac{\lambda_{0}}{\lambda_{1}} V_{1} V_{2}+\frac{\lambda_{0}}{\lambda_{1}} V_{1} V_{6}+\frac{\lambda_{0}^{2}}{\lambda_{1}^{2}} V_{1} V_{8}\right. \\
& \left.+V_{2}^{2}+V_{2} V_{6}+\frac{\lambda_{0}}{\lambda_{1}} V_{2} V_{8}+V_{2} V_{5}+V_{5} V_{6}+\frac{\lambda_{0}}{\lambda_{1}} V_{5} V_{8}\right) \\
& =\frac{k_{1} k_{2}}{\lambda_{1}^{2}}+\frac{k_{1} k_{5}}{\lambda_{1}^{2}}+\frac{k_{1}^{2}}{\lambda_{1}^{2}}+\frac{k_{2}^{2}+k_{2}}{\lambda_{1}^{2}}+\frac{k_{2} k_{5}}{\lambda_{1}^{2}}+\frac{k_{1} k_{2}}{\lambda_{1}^{2}}+\frac{k_{2} k_{5}}{\lambda_{1}^{2}}+\frac{k_{5}^{2}}{\lambda_{1}^{2}}+\frac{k_{1} k_{5}}{\lambda_{1}^{2}}=\frac{k_{2}}{\lambda_{1}^{2}}+1
\end{aligned}
$$

and consequently

$$
\operatorname{cov}\left(Z_{1}, Z_{3}\right)=\mathbf{E} Z_{1} Z_{2}-\mathbf{E} Z_{1} \mathbf{E} Z_{2}=\frac{k_{2}}{\lambda_{1}^{2}}
$$

Now we are able to derive the correlation:

$$
\rho_{1}=\frac{\operatorname{cov}\left(Z_{1}, Z_{3}\right)}{\sqrt{V\left(Z_{1}\right) V\left(Z_{3}\right)}}=\frac{k_{2}}{\lambda_{1}}=k_{2} \sigma_{1}^{2}
$$

Similar calculations imply $\rho_{2}=k_{3} \sigma_{2}^{2}$ and $\rho=k_{1} \sigma_{1} \sigma_{2}$. Consequently, $k_{1}+k_{2}+k_{5}=\frac{1}{\sigma_{1}^{2}}$ and $k_{1}+k_{3}+k_{4}=\frac{1}{\sigma_{2}^{2}}$ imply the following relations:

$k_{5}=\frac{1}{\sigma_{1}^{2}}-k_{2}-k_{1}=\frac{1}{\sigma_{1}^{2}}-\frac{\rho_{1}}{\sigma_{1}^{2}}-\frac{\rho}{\sigma_{1} \sigma_{2}} \quad$ and $\quad k_{4}=\frac{1}{\sigma_{2}^{2}}-k_{3}-k_{4}=\frac{1}{\sigma_{2}^{2}}-\frac{\rho_{2}}{\sigma_{2}^{2}}-\frac{\rho}{\sigma_{1} \sigma_{2}}$. If $Y \sim \Gamma(k, \lambda)$, then $\mathbf{E} e^{-s Y}=\left(1+\frac{s}{\lambda}\right)^{-k}$. Now we are in the state to derive the survival function in $(7)$ : 


$$
\begin{aligned}
S\left(x_{1}, y_{1}, x_{2}, y_{2}\right) & \\
= & \mathbf{E} S_{1}\left(x_{1}\right)^{Z_{1}} S_{2}\left(y_{1}\right)^{Z_{2}} S_{1}\left(x_{2}\right)^{Z_{3}} S_{2}\left(y_{2}\right)^{Z_{4}} \\
= & \mathbf{E} e^{-V_{1}\left(\frac{\lambda_{0}}{\lambda_{1}} H_{1}\left(x_{1}\right)+\frac{\lambda_{0}}{\lambda_{2}} H_{2}\left(y_{1}\right)\right)} e^{-V_{2}\left(H_{1}\left(x_{1}\right)+H_{1}\left(x_{2}\right)\right)} \\
* & e^{-V_{3}\left(H_{2}\left(y_{1}\right)+H_{2}\left(y_{2}\right)\right)} e^{-V_{8}\left(\frac{\lambda_{0}}{\lambda_{1}} H_{1}\left(x_{2}\right)+\frac{\lambda_{0}}{\lambda_{2}} H_{2}\left(y_{2}\right)\right)} e^{-V_{4} H_{2}\left(y_{1}\right)} e^{-V_{5} H_{1}\left(x_{1}\right)} e^{-V_{6} H_{1}\left(x_{2}\right)} e^{-V_{7} H_{2}\left(y_{2}\right)} \\
= & \left(1+\frac{1}{\lambda_{0}}\left(\frac{\lambda_{0}}{\lambda_{1}} H_{1}\left(x_{1}\right)+\frac{\lambda_{0}}{\lambda_{2}} H_{2}\left(y_{1}\right)\right)\right)^{-k_{1}}\left(1+\frac{1}{\lambda_{1}} H_{1}\left(x_{1}\right)+\frac{1}{\lambda_{1}} H_{1}\left(x_{2}\right)\right)^{-k_{2}} \\
& *\left(1+\frac{1}{\lambda_{2}} H_{2}\left(y_{1}\right)+\frac{1}{\lambda_{2}} H_{2}\left(y_{2}\right)\right)^{-k_{3}}\left(1+\frac{1}{\lambda_{0}}\left(\frac{\lambda_{0}}{\lambda_{1}} H_{1}\left(x_{2}\right)+\frac{\lambda_{0}}{\lambda_{2}} H_{2}\left(y_{2}\right)\right)\right)^{-k_{1}} \\
& *\left(1+\frac{1}{\lambda_{2}} H_{2}\left(y_{1}\right)\right)^{-k_{4}}\left(1+\frac{1}{\lambda_{1}} H_{1}\left(x_{1}\right)\right)^{-k_{5}}\left(1+\frac{1}{\lambda_{1}} H_{1}\left(x_{2}\right)\right)^{-k_{5}}\left(1+\frac{1}{\lambda_{2}} H_{2}\left(y_{2}\right)\right)^{-k_{4}} \\
= & \left(S_{1}\left(x_{1}\right)^{-\sigma_{1}^{2}}+S_{1}\left(x_{2}\right)^{-\sigma_{1}^{2}}-1\right)^{-\rho_{1} / \sigma_{1}^{2}}\left(S_{2}\left(y_{1}\right)^{-\sigma_{2}^{2}}+S_{2}\left(y_{2}\right)^{-\sigma_{2}^{2}}-1\right)^{-\rho_{2} / \sigma_{2}^{2}} \\
* & \left(S_{1}\left(x_{1}\right)^{-\sigma_{1}^{2}}+S_{2}\left(y_{1}\right)^{-\sigma_{2}^{2}}-1\right)^{-\rho / \sigma_{1} \sigma_{2}}\left(S_{1}\left(x_{2}\right)^{-\sigma_{1}^{2}}+S_{2}\left(y_{2}\right)^{-\sigma_{2}^{2}}-1\right)^{-\rho / \sigma_{1} \sigma_{2}} \\
* & S_{2}\left(y_{1}\right)^{1-\rho_{2}-\frac{\sigma_{2}}{\sigma_{1}} \rho} S_{1}\left(x_{1}\right)^{1-\rho_{1}-\frac{\sigma_{1}}{\sigma_{2}} \rho} S_{1}\left(x_{2}\right)^{1-\rho_{1}-\frac{\sigma_{1}}{\sigma_{2}} \rho} S_{2}\left(y_{2}\right)^{1-\rho_{2}-\frac{\sigma_{2}}{\sigma_{1}} \rho}
\end{aligned}
$$

The likelihood function (of the truncated data) is of the following form:

$$
\begin{aligned}
L\left(t_{1}, t_{2}, \delta_{1}, \delta_{2}, w\right) & =\left(1\left(\delta_{1}=1, \delta_{2}=1\right) S_{x_{1} x_{2}}\left(t_{1}, t_{1}, t_{2}, t_{2}\right)\right. \\
& +1\left(\delta_{1}=1, \delta_{2}=0\right) S_{x_{1}}\left(t_{1}, t_{1}, t_{2}, t_{2}\right) \\
& +1\left(\delta_{1}=0, \delta_{2}=1\right) S_{x_{2}}\left(t_{1}, t_{1}, t_{2}, t_{2}\right) \\
& +1\left(\delta_{1}=0, \delta_{2}=0\right) S\left(t_{1}, t_{1}, t_{2}, t_{2}\right) \\
& +1\left(\delta_{1}=-1, \delta_{2}=-1\right) S_{y_{1} y_{2}}\left(t_{1}, t_{1}, t_{2}, t_{2}\right) \\
& +1\left(\delta_{1}=-1, \delta_{2}=0\right) S_{y_{1}}\left(t_{1}, t_{1}, t_{2}, t_{2}\right) \\
& +1\left(\delta_{1}=0, \delta_{2}=-1\right) S_{y_{2}}\left(t_{1}, t_{1}, t_{2}, t_{2}\right) \\
& +1\left(\delta_{1}=1, \delta_{2}=-1\right) S_{x_{1} y_{2}}\left(t_{1}, t_{1}, t_{2}, t_{2}\right) \\
& \left.+1\left(\delta_{1}=-1, \delta_{2}=1\right) S_{y_{1} x_{2}}\left(t_{1}, t_{1}, t_{2}, t_{2}\right)\right) / S(w, w, w, w)
\end{aligned}
$$


The derivatives are given by

$$
\begin{aligned}
& S_{x_{1}}\left(x_{1}, y_{1}, x_{2}, y_{2}\right)=-\rho_{1}\left(S_{1}\left(x_{1}\right)^{-\sigma_{1}^{2}}+S_{1}\left(y_{1}\right)^{-\sigma_{1}^{2}}-1\right)^{-\frac{\rho_{1}}{\sigma_{1}^{2}}-1} \\
& \text { * }\left(S_{2}\left(x_{2}\right)^{-\sigma_{2}^{2}}+S_{2}\left(y_{2}\right)^{-\sigma_{2}^{2}}-1\right)^{-\frac{\rho_{2}}{\sigma_{2}^{2}}} \\
& \text { * }\left(S_{1}\left(x_{1}\right)^{-\sigma_{1}^{2}}+S_{2}\left(x_{2}\right)^{-\sigma_{2}^{2}}-1\right)^{-\frac{\rho}{\sigma_{1} \sigma_{2}}} \\
& \text { * }\left(S_{1}\left(y_{1}\right)^{-\sigma_{1}^{2}}+S_{2}\left(y_{2}\right)^{-\sigma_{2}^{2}}-1\right)^{-\frac{\rho}{\sigma_{1} \sigma_{2}}} \\
& \text { * } S_{1}\left(x_{1}\right)^{1-\rho_{1}-\frac{\sigma_{1}}{\sigma_{2}} \rho-\sigma_{1}^{2}} \mu_{1}\left(x_{1}\right) \\
& \text { * } S_{1}\left(y_{1}\right)^{1-\rho_{1}-\frac{\sigma_{1}}{\sigma_{2}} \rho} \\
& \text { * } S_{2}\left(x_{2}\right)^{1-\rho_{2}-\frac{\sigma_{2}}{\sigma_{1}} \rho} \\
& \text { * } S_{2}\left(y_{2}\right)^{1-\rho_{2}-\frac{\sigma_{2}}{\sigma_{1}} \rho} \\
& -\frac{\sigma_{1}}{\sigma_{2}} \rho\left(S_{1}\left(x_{1}\right)^{-\sigma_{1}^{2}}+S_{1}\left(y_{1}\right)^{-\sigma_{1}^{2}}-1\right)^{-\frac{\rho_{1}}{\sigma_{1}^{2}}} \\
& \text { * }\left(S_{2}\left(x_{2}\right)^{-\sigma_{2}^{2}}+S_{2}\left(y_{2}\right)^{-\sigma_{2}^{2}}-1\right)^{-\frac{\rho_{2}}{\sigma_{2}^{2}}} \\
& \text { * }\left(S_{1}\left(x_{1}\right)^{-\sigma_{1}^{2}}+S_{2}\left(x_{2}\right)^{-\sigma_{2}^{2}}-1\right)^{-\frac{\rho}{\sigma_{1} \sigma_{2}}-1} \\
& \text { * }\left(S_{1}\left(y_{1}\right)^{-\sigma_{1}^{2}}+S_{2}\left(y_{2}\right)^{-\sigma_{2}^{2}}-1\right)^{-\frac{\rho}{\sigma_{1} \sigma_{2}}} \\
& \text { * } S_{1}\left(x_{1}\right)^{1-\rho_{1}-\frac{\sigma_{1}}{\sigma_{2}} \rho-\sigma_{1}^{2}} \mu_{1}\left(x_{1}\right) \\
& \text { * } S_{1}\left(y_{1}\right)^{1-\rho_{1}-\frac{\sigma_{1}}{\sigma_{2}} \rho} \\
& \text { * } S_{2}\left(x_{2}\right)^{1-\rho_{2}-\frac{\sigma_{2}}{\sigma_{1}} \rho} \\
& \text { * } S_{2}\left(y_{2}\right)^{1-\rho_{2}-\frac{\sigma_{2}}{\sigma_{1}} \rho} \\
& -\left(1-\rho_{1}-\frac{\sigma_{1}}{\sigma_{2}} \rho\right) \sigma_{1}^{2}\left(S_{1}\left(x_{1}\right)^{-\sigma_{1}^{2}}+S_{1}\left(y_{1}\right)^{-\sigma_{1}^{2}}-1\right)^{-\frac{\rho_{1}}{\sigma_{1}^{2}}} \\
& \text { * }\left(S_{2}\left(x_{2}\right)^{-\sigma_{2}^{2}}+S_{2}\left(y_{2}\right)^{-\sigma_{2}^{2}}-1\right)^{-\frac{\rho_{2}}{\sigma_{2}^{2}}} \\
& \text { * }\left(S_{1}\left(x_{1}\right)^{-\sigma_{1}^{2}}+S_{2}\left(x_{2}\right)^{-\sigma_{2}^{2}}-1\right)^{-\frac{\rho}{\sigma_{1} \sigma_{2}}} \\
& \text { * }\left(S_{1}\left(y_{1}\right)^{-\sigma_{1}^{2}}+S_{2}\left(y_{2}\right)^{-\sigma_{2}^{2}}-1\right)^{-\frac{\rho}{\sigma_{1} \sigma_{2}}} \\
& \text { * } S_{1}\left(x_{1}\right)^{1-\rho_{1}-\frac{\sigma_{1}}{\sigma_{2}} \rho} \mu_{1}\left(x_{1}\right) S_{1}\left(y_{1}\right)^{1-\rho_{1}-\frac{\sigma_{1}}{\sigma_{2}} \rho} \\
& \text { * } S_{2}\left(x_{2}\right)^{1-\rho_{2}-\frac{\sigma_{2}}{\sigma_{1}} \rho} S_{2}\left(y_{2}\right)^{1-\rho_{2}-\frac{\sigma_{2}}{\sigma_{1}} \rho}
\end{aligned}
$$




$$
\begin{aligned}
& S_{y_{1}}\left(x_{1}, y_{1}, x_{2}, y_{2}\right)=-\rho_{1}\left(S_{1}\left(x_{1}\right)^{-\sigma_{1}^{2}}+S_{1}\left(y_{1}\right)^{-\sigma_{1}^{2}}-1\right)^{-\frac{\rho_{1}}{\sigma_{1}^{2}}-1} \\
& \text { * }\left(S_{2}\left(x_{2}\right)^{-\sigma_{2}^{2}}+S_{2}\left(y_{2}\right)^{-\sigma_{2}^{2}}-1\right)^{-\frac{\rho_{2}}{\sigma_{2}^{2}}} \\
& \text { * }\left(S_{1}\left(x_{1}\right)^{-\sigma_{1}^{2}}+S_{2}\left(x_{2}\right)^{-\sigma_{2}^{2}}-1\right)^{-\frac{\rho}{\sigma_{1} \sigma_{2}}} \\
& \text { * }\left(S_{1}\left(y_{1}\right)^{-\sigma_{1}^{2}}+S_{2}\left(y_{2}\right)^{-\sigma_{2}^{2}}-1\right)^{-\frac{\rho}{\sigma_{1} \sigma_{2}}} \\
& \text { * } S_{1}\left(x_{1}\right)^{1-\rho_{1}-\frac{\sigma_{1}}{\sigma_{2}} \rho} \\
& \text { * } S_{1}\left(y_{1}\right)^{1-\rho_{1}-\frac{\sigma_{1}}{\sigma_{2}} \rho-\sigma_{1}^{2}} \mu_{1}\left(y_{1}\right) \\
& \text { * } S_{2}\left(x_{2}\right)^{1-\rho_{2}-\frac{\sigma_{2}}{\sigma_{1}} \rho} \\
& \text { * } S_{2}\left(y_{2}\right)^{1-\rho_{2}-\frac{\sigma_{2}}{\sigma_{1}} \rho} \\
& -\frac{\sigma_{1}}{\sigma_{2}} \rho\left(S_{1}\left(x_{1}\right)^{-\sigma_{1}^{2}}+S_{1}\left(y_{1}\right)^{-\sigma_{1}^{2}}-1\right)^{-\frac{\rho_{1}}{\sigma_{1}^{2}}} \\
& \text { * }\left(S_{2}\left(x_{2}\right)^{-\sigma_{2}^{2}}+S_{2}\left(y_{2}\right)^{-\sigma_{2}^{2}}-1\right)^{-\frac{\rho_{2}}{\sigma_{2}^{2}}} \\
& \text { * }\left(S_{1}\left(x_{1}\right)^{-\sigma_{1}^{2}}+S_{2}\left(x_{2}\right)^{-\sigma_{2}^{2}}-1\right)^{-\frac{\rho}{\sigma_{1} \sigma_{2}}} \\
& \text { * }\left(S_{1}\left(y_{1}\right)^{-\sigma_{1}^{2}}+S_{2}\left(y_{2}\right)^{-\sigma_{2}^{2}}-1\right)^{-\frac{\rho}{\sigma_{1} \sigma_{2}}-1} \\
& \text { * } S_{1}\left(x_{1}\right)^{1-\rho_{1}-\frac{\sigma_{1}}{\sigma_{2}} \rho} \\
& \text { * } S_{1}\left(y_{1}\right)^{1-\rho_{1}-\frac{\sigma_{1}}{\sigma_{2}} \rho-\sigma_{1}^{2}} \mu_{1}\left(y_{1}\right) \\
& \text { * } S_{2}\left(x_{2}\right)^{1-\rho_{2}-\frac{\sigma_{2}}{\sigma_{1}} \rho} \\
& \text { * } S_{2}\left(y_{2}\right)^{1-\rho_{2}-\frac{\sigma_{2}}{\sigma_{1}} \rho} \\
& \text { - }\left(1-\rho_{1}-\frac{\sigma_{1}}{\sigma_{2}} \rho\right) \sigma_{1}^{2}\left(S_{1}\left(x_{1}\right)^{-\sigma_{1}^{2}}+S_{1}\left(y_{1}\right)^{-\sigma_{1}^{2}}-1\right)^{-\frac{\rho_{1}}{\sigma_{1}^{2}}} \\
& \text { * }\left(S_{2}\left(x_{2}\right)^{-\sigma_{2}^{2}}+S_{2}\left(y_{2}\right)^{-\sigma_{2}^{2}}-1\right)^{-\frac{\rho_{2}}{\sigma_{2}^{2}}} \\
& \text { * }\left(S_{1}\left(x_{1}\right)^{-\sigma_{1}^{2}}+S_{2}\left(x_{2}\right)^{-\sigma_{2}^{2}}-1\right)^{-\frac{\rho}{\sigma_{1} \sigma_{2}}} \\
& \text { * }\left(S_{1}\left(y_{1}\right)^{-\sigma_{1}^{2}}+S_{2}\left(y_{2}\right)^{-\sigma_{2}^{2}}-1\right)^{-\frac{\rho}{\sigma_{1} \sigma_{2}}} \\
& \text { * } S_{1}\left(x_{1}\right)^{1-\rho_{1}-\frac{\sigma_{1}}{\sigma_{2}} \rho} \\
& \text { * } S_{1}\left(y_{1}\right)^{1-\rho_{1}-\frac{\sigma_{1}}{\sigma_{2}} \rho} \mu_{1}\left(y_{1}\right) \\
& \text { * } S_{2}\left(x_{2}\right)^{1-\rho_{2}-\frac{\sigma_{2}}{\sigma_{1}} \rho} \\
& \text { * } S_{2}\left(y_{2}\right)^{1-\rho_{2}-\frac{\sigma_{2}}{\sigma_{1}} \rho}
\end{aligned}
$$




$$
\begin{aligned}
& S_{x_{2}}\left(x_{1}, y_{1}, x_{2}, y_{2}\right)=-\rho_{2}\left(S_{1}\left(x_{1}\right)^{-\sigma_{1}^{2}}+S_{1}\left(y_{1}\right)^{-\sigma_{1}^{2}}-1\right)^{-\frac{\rho_{1}}{\sigma_{1}^{2}}} \\
& \text { * }\left(S_{2}\left(x_{2}\right)^{-\sigma_{2}^{2}}+S_{2}\left(y_{2}\right)^{-\sigma_{2}^{2}}-1\right)^{-\frac{\rho_{2}}{\sigma_{2}^{2}}-1} \\
& \text { * }\left(S_{1}\left(x_{1}\right)^{-\sigma_{1}^{2}}+S_{2}\left(x_{2}\right)^{-\sigma_{2}^{2}}-1\right)^{-\frac{\rho}{\sigma_{1} \sigma_{2}}} \\
& \text { * }\left(S_{1}\left(y_{1}\right)^{-\sigma_{1}^{2}}+S_{2}\left(y_{2}\right)^{-\sigma_{2}^{2}}-1\right)^{-\frac{\rho}{\sigma_{1} \sigma_{2}}} \\
& \text { * } S_{1}\left(x_{1}\right)^{1-\rho_{1}-\frac{\sigma_{1}}{\sigma_{2}} \rho} \\
& \text { * } S_{1}\left(y_{1}\right)^{1-\rho_{1}-\frac{\sigma_{1}}{\sigma_{2}} \rho} \\
& \text { * } S_{2}\left(x_{2}\right)^{1-\rho_{2}-\frac{\sigma_{2}}{\sigma_{1}} \rho-\sigma_{2}^{2}} \mu_{2}\left(x_{2}\right) \\
& \text { * } S_{2}\left(y_{2}\right)^{1-\rho_{2}-\frac{\sigma_{2}}{\sigma_{1}} \rho} \\
& -\frac{\sigma_{2}}{\sigma_{1}} \rho\left(S_{1}\left(x_{1}\right)^{-\sigma_{1}^{2}}+S_{1}\left(y_{1}\right)^{-\sigma_{1}^{2}}-1\right)^{-\frac{\rho_{1}}{\sigma_{1}^{2}}} \\
& \text { * }\left(S_{2}\left(x_{2}\right)^{-\sigma_{2}^{2}}+S_{2}\left(y_{2}\right)^{-\sigma_{2}^{2}}-1\right)^{-\frac{\rho_{2}}{\sigma_{2}^{2}}} \\
& \text { * }\left(S_{1}\left(x_{1}\right)^{-\sigma_{1}^{2}}+S_{2}\left(x_{2}\right)^{-\sigma_{2}^{2}}-1\right)^{-\frac{\rho}{\sigma_{1} \sigma_{2}}-1} \\
& \text { * }\left(S_{1}\left(y_{1}\right)^{-\sigma_{1}^{2}}+S_{2}\left(y_{2}\right)^{-\sigma_{2}^{2}}-1\right)^{-\frac{\rho}{\sigma_{1} \sigma_{2}}} \\
& \text { * } S_{1}\left(x_{1}\right)^{1-\rho_{1}-\frac{\sigma_{1}}{\sigma_{2}} \rho} \\
& \text { * } S_{1}\left(y_{1}\right)^{1-\rho_{1}-\frac{\sigma_{1}}{\sigma_{2}} \rho} \\
& \text { * } S_{2}\left(x_{2}\right)^{1-\rho_{2}-\frac{\sigma_{2}}{\sigma_{1}} \rho-\sigma_{2}^{2}} \mu_{2}\left(x_{2}\right) \\
& \text { * } S_{2}\left(y_{2}\right)^{1-\rho_{2}-\frac{\sigma_{2}}{\sigma_{1}} \rho} \\
& -\left(1-\rho_{2}-\frac{\sigma_{2}}{\sigma_{1}} \rho\right) \sigma_{2}^{2}\left(S_{1}\left(x_{1}\right)^{-\sigma_{1}^{2}}+S_{1}\left(y_{1}\right)^{-\sigma_{1}^{2}}-1\right)^{-\frac{\rho_{1}}{\sigma_{1}^{2}}} \\
& \text { * }\left(S_{2}\left(x_{2}\right)^{-\sigma_{2}^{2}}+S_{2}\left(y_{2}\right)^{-\sigma_{2}^{2}}-1\right)^{-\frac{\rho_{2}}{\sigma_{2}^{2}}} \\
& \text { * }\left(S_{1}\left(x_{1}\right)^{-\sigma_{1}^{2}}+S_{2}\left(x_{2}\right)^{-\sigma_{2}^{2}}-1\right)^{-\frac{\rho}{\sigma_{1} \sigma_{2}}} \\
& \text { * }\left(S_{1}\left(y_{1}\right)^{-\sigma_{1}^{2}}+S_{2}\left(y_{2}\right)^{-\sigma_{2}^{2}}-1\right)^{-\frac{\rho}{\sigma_{1} \sigma_{2}}} \\
& \text { * } S_{1}\left(x_{1}\right)^{1-\rho_{1}-\frac{\sigma_{1}}{\sigma_{2}} \rho} \\
& \text { * } S_{1}\left(y_{1}\right)^{1-\rho_{1}-\frac{\sigma_{1}}{\sigma_{2}} \rho} \\
& \text { * } S_{2}\left(x_{2}\right)^{1-\rho_{2}-\frac{\sigma_{2}}{\sigma_{1}} \rho} \mu_{2}\left(x_{2}\right) \\
& \text { * } S_{2}\left(y_{2}\right)^{1-\rho_{2}-\frac{\sigma_{2}}{\sigma_{1}} \rho}
\end{aligned}
$$




$$
\begin{aligned}
& S_{y_{2}}\left(x_{1}, y_{1}, x_{2}, y_{2}\right)=-\rho_{2}\left(S_{1}\left(x_{1}\right)^{-\sigma_{1}^{2}}+S_{1}\left(y_{1}\right)^{-\sigma_{1}^{2}}-1\right)^{-\frac{\rho_{1}}{\sigma_{1}^{2}}} \\
& \text { * }\left(S_{2}\left(x_{2}\right)^{-\sigma_{2}^{2}}+S_{2}\left(y_{2}\right)^{-\sigma_{2}^{2}}-1\right)^{-\frac{\rho_{2}}{\sigma_{2}^{2}}-1} \\
& \text { * }\left(S_{1}\left(x_{1}\right)^{-\sigma_{1}^{2}}+S_{2}\left(x_{2}\right)^{-\sigma_{2}^{2}}-1\right)^{-\frac{\rho}{\sigma_{1} \sigma_{2}}} \\
& \text { * }\left(S_{1}\left(y_{1}\right)^{-\sigma_{1}^{2}}+S_{2}\left(y_{2}\right)^{-\sigma_{2}^{2}}-1\right)^{-\frac{\rho}{\sigma_{1} \sigma_{2}}} \\
& \text { * } S_{1}\left(x_{1}\right)^{1-\rho_{1}-\frac{\sigma_{1}}{\sigma_{2}} \rho} \\
& \text { * } S_{1}\left(y_{1}\right)^{1-\rho_{1}-\frac{\sigma_{1}}{\sigma_{2}} \rho} \\
& \text { * } \quad S_{2}\left(x_{2}\right)^{1-\rho_{2}-\frac{\sigma_{2}}{\sigma_{1}} \rho} \\
& \text { * } S_{2}\left(y_{2}\right)^{1-\rho_{2}-\frac{\sigma_{2}}{\sigma_{1}} \rho-\sigma_{2}^{2}} \mu_{2}\left(y_{2}\right) \\
& -\frac{\sigma_{1}}{\sigma_{2}} \rho\left(S_{1}\left(x_{1}\right)^{-\sigma_{1}^{2}}+S_{1}\left(y_{1}\right)^{-\sigma_{1}^{2}}-1\right)^{-\frac{\rho_{1}}{\sigma_{1}^{2}}} \\
& \text { * }\left(S_{2}\left(x_{2}\right)^{-\sigma_{2}^{2}}+S_{2}\left(y_{2}\right)^{-\sigma_{2}^{2}}-1\right)^{-\frac{\rho_{2}}{\sigma_{2}^{2}}} \\
& \text { * }\left(S_{1}\left(x_{1}\right)^{-\sigma_{1}^{2}}+S_{2}\left(x_{2}\right)^{-\sigma_{2}^{2}}-1\right)^{-\frac{\rho}{\sigma_{1} \sigma_{2}}} \\
& \text { * }\left(S_{1}\left(y_{1}\right)^{-\sigma_{1}^{2}}+S_{2}\left(y_{2}\right)^{-\sigma_{2}^{2}}-1\right)^{-\frac{\rho}{\sigma_{1} \sigma_{2}}-1} \\
& \text { * } \quad S_{1}\left(x_{1}\right)^{1-\rho_{1}-\frac{\sigma_{1}}{\sigma_{2}} \rho} \\
& \text { * } S_{1}\left(y_{1}\right)^{1-\rho_{1}-\frac{\sigma_{1}}{\sigma_{2}} \rho} \\
& \text { * } \quad S_{2}\left(x_{2}\right)^{1-\rho_{2}-\frac{\sigma_{2}}{\sigma_{1}} \rho} \\
& \text { * } S_{2}\left(y_{2}\right)^{1-\rho_{2}-\frac{\sigma_{2}}{\sigma_{1}} \rho-\sigma_{2}^{2}} \mu_{2}\left(y_{2}\right) \\
& \text { - }\left(1-\rho_{2}-\frac{\sigma_{2}}{\sigma_{1}} \rho\right) \sigma_{2}^{2}\left(S_{1}\left(x_{1}\right)^{-\sigma_{1}^{2}}+S_{1}\left(y_{1}\right)^{-\sigma_{1}^{2}}-1\right)^{-\frac{\rho_{1}}{\sigma_{1}^{2}}} \\
& \text { * }\left(S_{2}\left(x_{2}\right)^{-\sigma_{2}^{2}}+S_{2}\left(y_{2}\right)^{-\sigma_{2}^{2}}-1\right)^{-\frac{\rho_{2}}{\sigma_{2}^{2}}} \\
& \text { * }\left(S_{1}\left(x_{1}\right)^{-\sigma_{1}^{2}}+S_{2}\left(x_{2}\right)^{-\sigma_{2}^{2}}-1\right)^{-\frac{\rho}{\sigma_{1} \sigma_{2}}} \\
& \text { * }\left(S_{1}\left(y_{1}\right)^{-\sigma_{1}^{2}}+S_{2}\left(y_{2}\right)^{-\sigma_{2}^{2}}-1\right)^{-\frac{\rho}{\sigma_{1} \sigma_{2}}} \\
& \text { * } S_{1}\left(x_{1}\right)^{1-\rho_{1}-\frac{\sigma_{1}}{\sigma_{2}} \rho} \\
& \text { * } S_{1}\left(y_{1}\right)^{1-\rho_{1}-\frac{\sigma_{1}}{\sigma_{2}} \rho} \\
& \text { * } S_{2}\left(x_{2}\right)^{1-\rho_{2}-\frac{\sigma_{2}}{\sigma_{1}} \rho} \\
& \text { * } S_{2}\left(y_{2}\right)^{1-\rho_{2}-\frac{\sigma_{2}}{\sigma_{1}} \rho} \mu_{2}\left(y_{2}\right)
\end{aligned}
$$




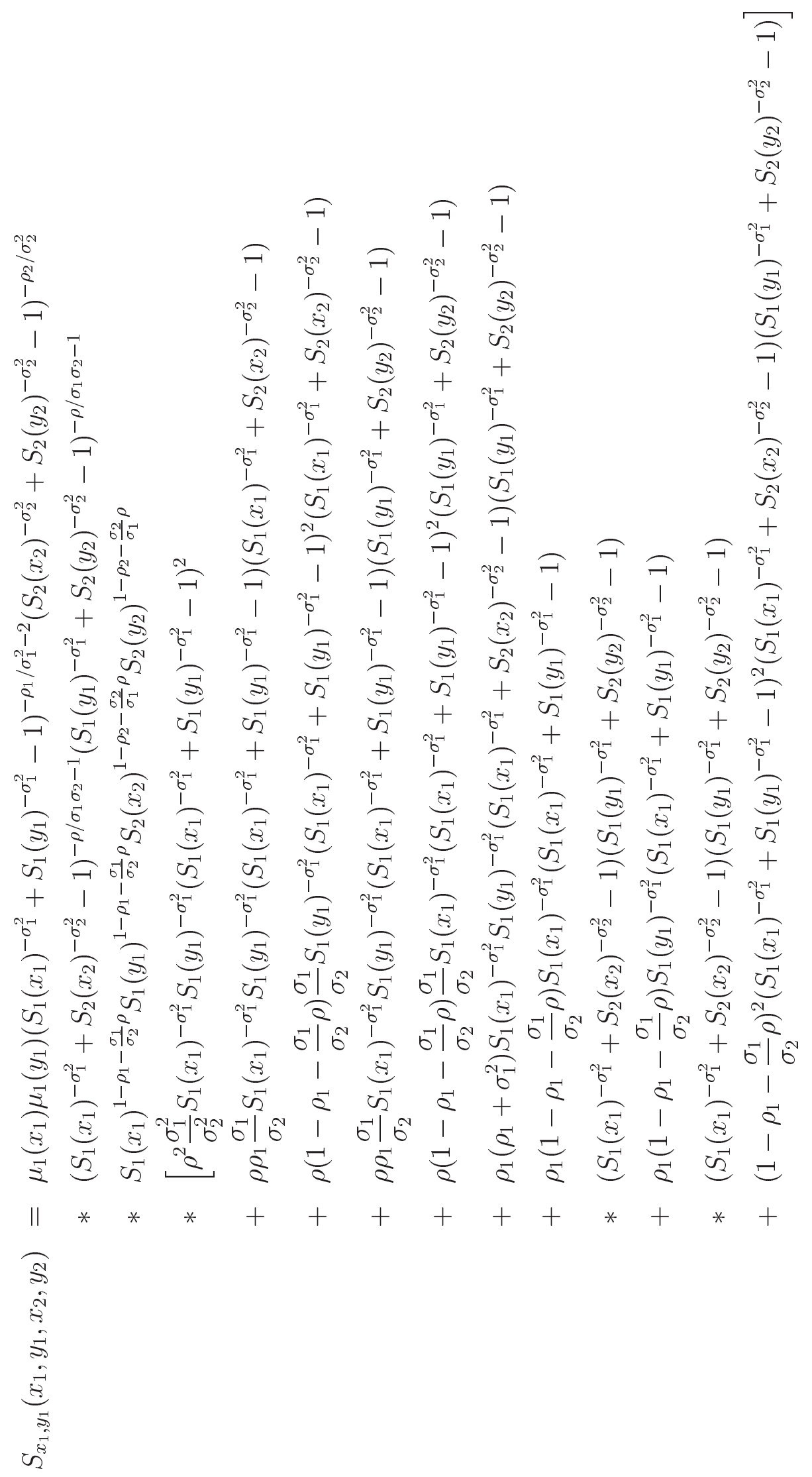




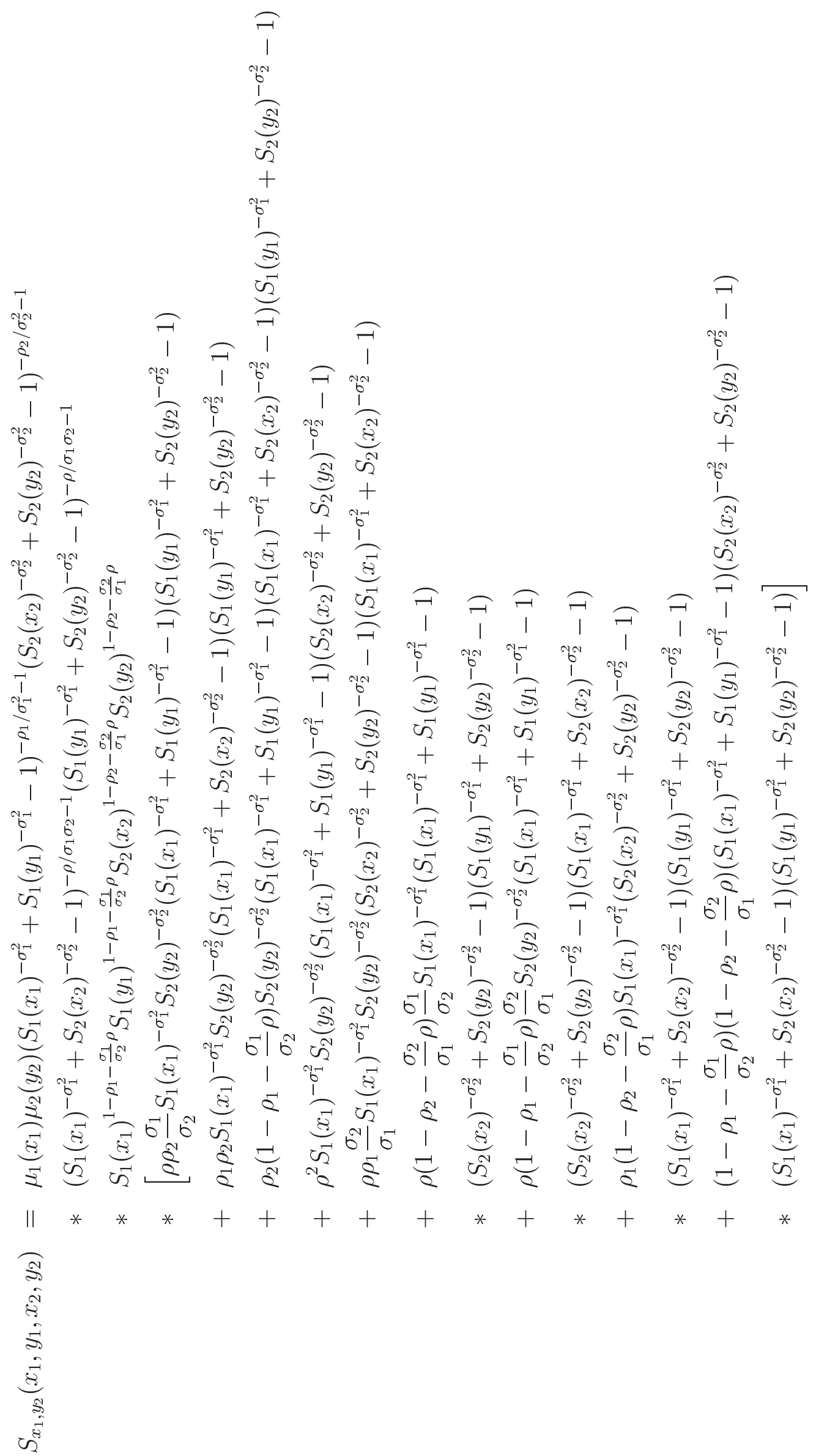




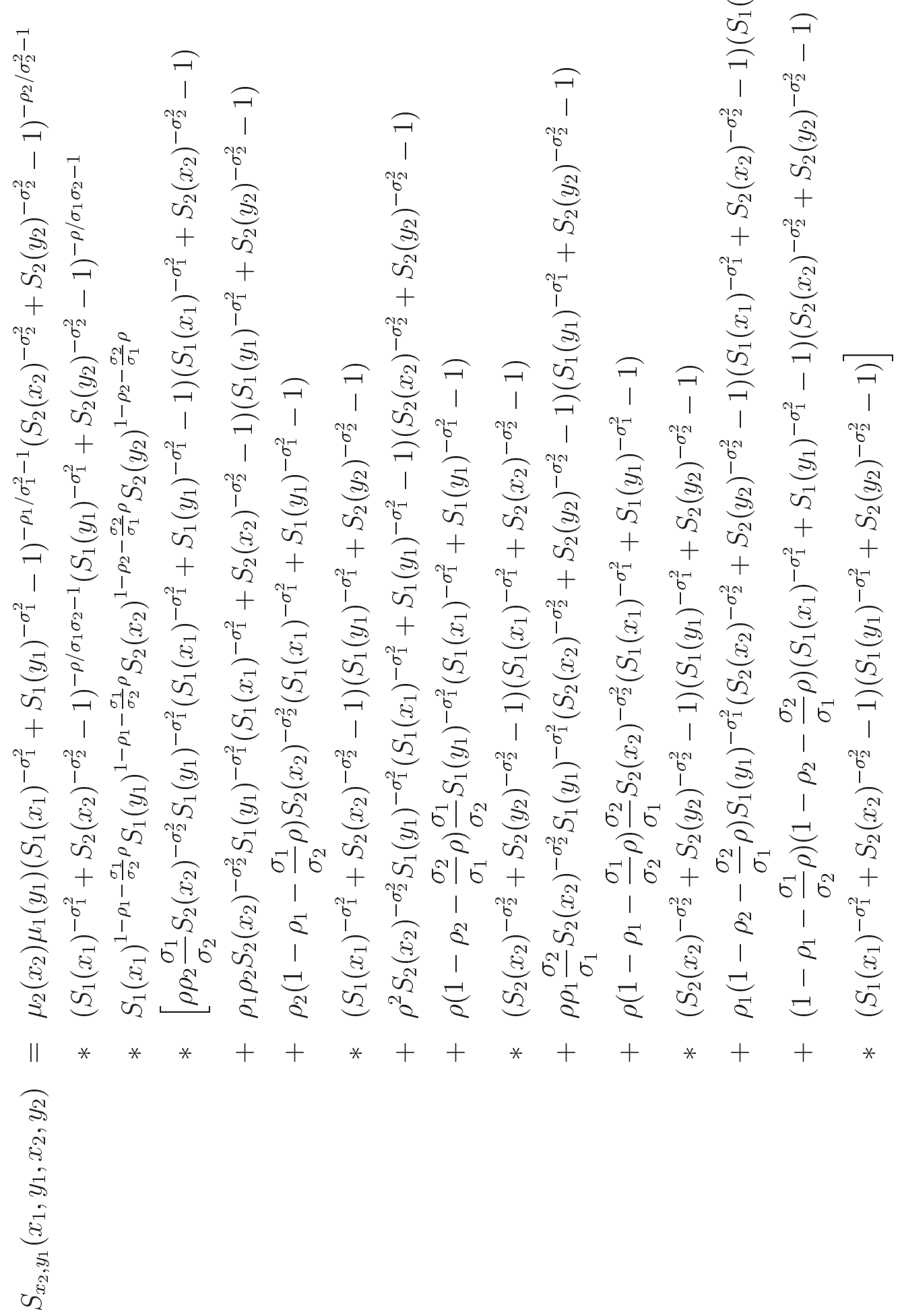




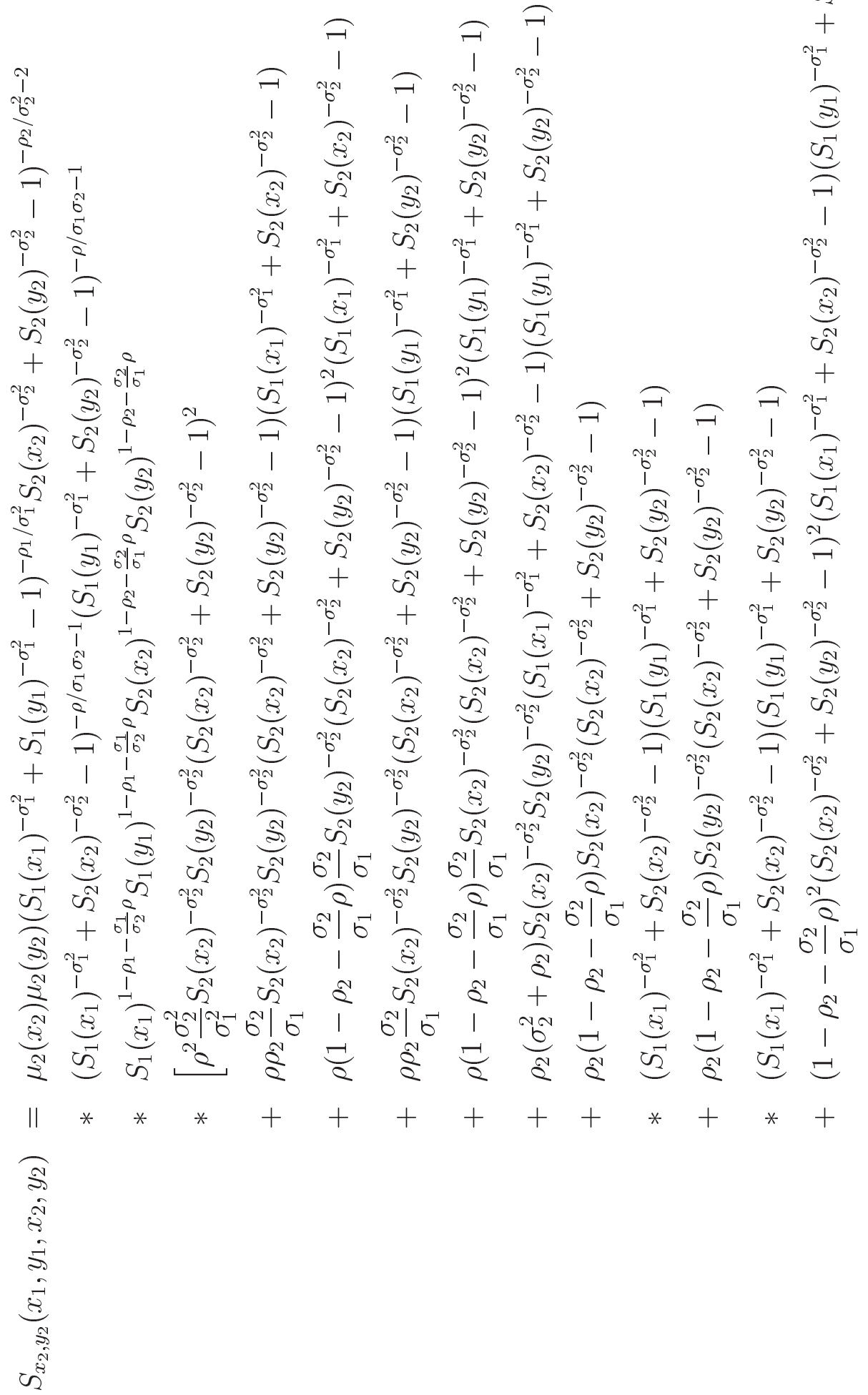

